\title{
Valeur alimentaire des marcs de raisin IV. Effet de différents niveaux de concentré de complémentation sur la digestibilité chez le mouton de rations de marc traité ou non à la soude
}

\author{
A. LARWENCE, Fatma HAMMOUDA et Yamina GAOUAS \\ avec la collaboration technique de Samia ABADA et Nacéra OUCHAI \\ Institut National Agronomique, Département de Zootechnie \\ El-Harrach, Alger
}

\begin{abstract}
Résumé
Pour déterminer la dose optimum d'un concentré à base de tourteau de colza qui assure la digestibilité maximale de l'ensilage de marcs de raisin traités ou non à la soude (à la dose de 0,67 et 1,11 p. 100 par rapport à la MS), nous avons mesuré sur des lots de quatre moutons adultes la digestibilité de rations renfermant $200,300,400$ et $500 \mathrm{~g}$ de concentré (soit par rapport à la MS respectivement $15,21,26$ et 31 p. 100 de la ration totale).

Les marcs de raisin se conservent par ensilage de façon satisfaisante. Lorsqu'ils sont traités à la soude, la production d'acide butyrique augmente de 12 points pour la dose de 1,11 p. 100 sans que la production d'ammoniac soit toutefois modifiée (tabl. 1). La digestibilité de la matière organique ( $\mathrm{dMO}$ ) progresse sous l'action du traitement; elle se situe à 32,42 et 40 p. 100 respectivement pour la dose de $0,0,67$ et 1,11 p. 100 de soude (tabl. 2).

La complémentation azotée et énergétique de ces trois catégories de marc n'exerce pas la même action sur la dMO de la ration totale et sur celle propre des marcs selon que le produit est traité ou non (fig. 1 et 2).

Ainsi, lorsque les mares ne sont pas traités, la dMO de la ration totale atteint un maximum pour la dose de $300 \mathrm{~g}$ de concentré puis reste inchangée par suite de la diminution de la dMO propre des marcs (qui passe de 41 à 35 et à 34 p. 100 respectivement pour la dose de 300,400 et $500 \mathrm{~g}$ de concentré).

Avec les marcs traités, la dMO de la ration totale augmente parallèlement à la dose de concentré. Par exemple, pour la dose de 0,67 p. 100 de soude, la dMO de la ration totale passe de 42 à 54 p. 100 , ce qui correspond globalement au maintien de la dMO propre des marcs traités. Il n'y aurait donc pas d'intéractions digestives significatives pour les marcs traités contrairement à ce qui est observé pour les marcs non traités.
\end{abstract}

Mots clés : Ensilage marc de raisin, niveau de concentré, traitement à la soude, digestibilité mouton. 


\section{Introduction}

Une revue de l'ensemble des travaux effectués sur les marcs de raisin depuis la première étude de FABRE en 1909 (LARWENCE, 1983) montre que la digestibilité de la matière organique et celle de l'azote se situent respectivement à 31 p. $100 \pm 8$ et à 15 p. $100 \pm 6$.

Les récents travaux de Messaoudi (1982) et de LARWENCE (1983) montrent que la faible digestibilité du produit résulte de l'action combinée de trois facteurs :

Une forte teneur en composés pariétaux (75 p. 100 de la MS) et en tanins ( 5 p. 100 de la MS) et une proportion élevée d'azote insoluble dans une solution tampon (91 p. 100). Les tanins agissent d'une part indirectement en insolubilisant l'azote du produit et d'autre part directement, en inhibant partiellement l'activité des microorganismes du rumen.

Le traitement à la soude des marcs de raisin à la dose de 0,67 p. 100 de la MS permet de diminuer l'effet inhibiteur des tanins et d'augmenter la proportion d'azote solubilisé dans le rumen; la digestibilité de la matière organique passe alors de 32 à 44 p. 100 (Larwence, Hammouda \& Gaouas, 1983).

L'augmentation de la proportion d'azote soluble de la ration peut être obtenue soit par une addition d'urée $(12 \mathrm{~g} / \mathrm{kg}$ de MS), soit par un apport de concentré. Dans ce dernier cas, sur 8 types de concentré isoazotés et isoénergétiques testés (LARWENCE \& Yahiaour, 1983), le mélange tourteau de colza-orge s'est révélé le plus efficace.

Dans le présent travail, nous étudions l'influence de différentes doses de ce concentré sur la digestibilité de rations composées de trois catégories de marcs : marcs non traités et marcs traités à la dose de 0,67 ou 1,11 p. 100 de soude par rapport à la MS.

\section{Matériel et Méthode}

\section{A. Mesure de la digestibilité des rations}

La méthode de mesure de la digestibilité, les analyses chimiques, la méthode de traitement des marcs à la soude ainsi que la préparation des ensilages ont été décrites par Larwence \& Yahiaoui (1983) ; Larwence, Hammouda \& Gaouas (1983). Dans le présent essai, les marcs de raisin sont distribués à raison de $1310 \mathrm{~g}$ de MS (pour le lot non complémenté) et de $860 \mathrm{~g}$ (pour les lots complémentés) par jour et par animal pesant en moyenne $42 \mathrm{~kg} \pm 2,5$; aucun refus n'est enregistré.

Le concentré de complémentation (62 p. 100 d'orge; 33 p. 100 de tourteau de colza ; 5 p. 100 de CMV) apporté à la dose de 200, 300, 400 et $500 \mathrm{~g}$ (soit respectivement $15,21,26$ et 31 p. 100 de la MS totale ingérée) par jour, est intimement mélangé aux marcs de raisin.

La digestibilité du concentré est calculée à partir des données des tables I.N.R.A. (1978). 


\section{B. Appréciation de la qualité des ensilages}

Le dosage des acides gras volatils (AGV) est effectué par chromatographie en phase gazeuse selon Rigaud \& Journet (1970). Les alcools sont dosés dans les mêmes conditions que les $\mathrm{AGV}$ à l'exception toutefois de la température de la chambre d'injection qui est réglée à $90^{\circ} \mathrm{C}$. Quant à l'ammoniac, il est dosé selon la méthode de Conway (1965).

La MS des ensilages est corrigée en appliquant l'équation de ScHoch (1949) modifiée par l'Institut technique des céréales et des fourrages pour la prise en compte à 100 p. 100 des alcools (Mosnier \& Papin, 1978).

\section{Résultats}

\section{A. Bilan des silos et composition chimique des marcs}

Les pertes totales par pourriture (pesée des marcs pourris) s'élèvent à 4,5 p. 100 pour les marcs non traités (MNT) et respectivement à 3,3 et 2,0 p. 100 pour les marcs traités à 0,67 p. 100 de soude (MT 0,67) et 1,11 p. 100 (MT 1,11). Bien que l'importance des moisissures soit liée à l'étanchéité à l'air des silos, la présence de soude semble néanmoins limiter la pourriture de surface.

Les paramètres fermentaires et la composition chimique des ensilages sont rapportés dans le tableau 1. La teneur en alcool des ensilages est faible; elle ne semble pas très nettement affectée par le traitement, à l'exception toutefois du propanol qui diminue lorsque la dose de soude augmente.

En revanche, sous l'effet du traitement, la teneur en acide acétique (C2) et en acide butyrique (C4) augmente dans de fortes proportions : C2 passe de $19 \mathrm{~g} / \mathrm{kg}$ de MS pour MNT à 26 et à $31 \mathrm{~g}$ respectivement pour MT 0,67 et MT 1,11 . Pour C4, l'augmentation est encore plus spectaculaire puisque sa teneur passe de $1,2 \mathrm{~g} / \mathrm{kg}$ de MS pour MNT à $12 \mathrm{~g}$ pour MT 1,11. A cette dose de soude apparaît par ailleurs de l'acide propionique.

Le pH est bas pour MNT $(3,17)$ et augmente avec la dose de soude. Mais, il reste néanmoins à un niveau relativement faible $(5,03$ et 5,77).

La composition chimique des marcs de raisin non traités n'appelle pas de commentaires particuliers : elle est en accord avec celle rapportée par nous-mêmes dans un précédent article (LARWENCE \& YaHIAOUI, 1983).

Le traitement modifie certains constituants : la teneur en matières azotées diminue par suite de pertes d'azote dans le jus d'ensilage (résultant du traitement par la solution de soude); elle passe de 15 p. 100 à 13 p. 100 respectivement pour MNT et MT 1,11 (tabl. 1).

L'augmentation de la teneur en cendres est normale et celle de la cellulose brute résulte soit de la formation de corps insolubles entre protéines et composés phénoliques [la formation de ces «artefacts » est particulièrement favorisée dans les marcs de raisin après traitement à la soude (HammoudA, 1984)] soit de l'accroissement des pertes en matière sèche sous forme de $\mathrm{C} 2$ et de $\mathrm{C} 4$. 


\section{TABLeaU 1}

Composition chimique (en p. 100 MS corrigée) et caractéristiques fermentaires de l'ensilage de marc.

Chemical composition (p. 100 DM corrected) and fermentation characteristics of grape marc silage.

\begin{tabular}{|c|c|c|c|}
\hline & MNT & MT 0,67 & MT 1,11 \\
\hline $\begin{array}{l}\text { Acide acétique }(\mathrm{g} / \mathrm{kg} \text { MS }) \ldots \ldots \ldots \\
\text { Acetic acid }(g / k g \text { DM })\end{array}$ & $18,7 \pm 1,2$ & $25,8 \pm 2,1$ & $31,5 \pm 1,9$ \\
\hline $\begin{array}{l}\text { Acide propionique }(\mathrm{g} / \mathrm{kg} \text { MS }) \ldots \ldots \\
\text { Propionic acid }(\mathrm{g} / \mathrm{kg} \text { DM })\end{array}$ & 0 & 0 & $1,3 \pm 0,04$ \\
\hline $\begin{array}{l}\text { Acide butyrique }(\mathrm{g} / \mathrm{kg} \text { MS }) \ldots \ldots \ldots \\
\text { Butyric acid }(g / \mathrm{kg} D M)\end{array}$ & $1,2 \pm 1,1$ & $1,7 \pm 0,3$ & $11,8 \pm 2,2$ \\
\hline $\begin{array}{l}\text { Méthanol }(\mathrm{g} / \mathrm{kg} \text { MS) } \ldots \ldots \ldots \ldots \ldots \ldots \\
\text { Methanol }(g / \mathrm{kg} D M)\end{array}$ & $0,32 \pm 0,11$ & $0,11 \pm 0,05$ & $0,20 \pm 0,01$ \\
\hline $\begin{array}{l}\text { Ethanol }(\mathrm{g} / \mathrm{kg} \text { MS }) \ldots \ldots \\
\text { Ethanol }(g / k g \quad D M)\end{array}$ & $0,65 \pm 0,32$ & $1,31 \pm 0,08$ & $0,53 \pm 0,04$ \\
\hline $\begin{array}{l}\text { Propanol }(\mathrm{g} / \mathrm{kg} \text { MS) } \ldots \ldots \ldots \ldots \\
\text { Propanol }(\mathrm{g} / \mathrm{kg} \text { DM) }\end{array}$ & $2,4 \pm 1,4$ & $0,72 \pm 0,10$ & $0,63 \pm 0,07$ \\
\hline $\mathrm{pH} \ldots \ldots \ldots \ldots \ldots \ldots$ & $3,14 \pm 1,30$ & $5,03 \pm 0,60$ & $5,77 \pm 0,80$ \\
\hline $\begin{array}{l}\mathrm{N}-\mathrm{NH}:(\% \quad \mathrm{~N} \text { total }) \ldots \ldots \ldots \ldots \ldots \ldots \\
N H_{s}-N \quad(\% \text { total nitrogen })\end{array}$ & $1,2 \pm 0,11$ & $2,9 \pm 0,21$ & $3,8 \pm 0,9$ \\
\hline $\begin{array}{l}\text { MS corrigée } \ldots \ldots \ldots \ldots \\
D M \text { corrected }\end{array}$ & 43,7 & 43,3 & 42,18 \\
\hline $\begin{array}{l}\text { Matières azotées }(\mathrm{N} \times 6,25) \\
\text { Crude protein }\end{array}$ & 15,3 & 13,8 & 13,0 \\
\hline Cellulose Weende. & 27,0 & 29,9 & 31,3 \\
\hline $\begin{array}{l}\text { Matières grasses } \ldots \ldots \\
\text { Ether extract }\end{array}$ & 7,8 & 8,2 & 7,7 \\
\hline $\begin{array}{l}\text { Cendres } \\
\text { Ash }\end{array}$ & 5,6 & 7,2 & 7,9 \\
\hline
\end{tabular}

B. Effet du niveau de concentré sur la digestibilité propre des marcs et sur celle de la ration totale

\section{Effet sur la digestibilité de la ration totale à base de marcs non traités}

Tous les principes nutritifs des rations (cellulose brute, matières azotées et matière grasse) voient leur digestibilité améliorée (tabl. 2). Celle de la matière organique s'établit à $32,42,51,49$ et 51 p. 100 respectivement pour la dose de $0,200,300,400$ et $500 \mathrm{~g}$ de concentré de complémentation. L'accroissement est important entre 0 et $200 \mathrm{~g}$ (10 points) et entre 200 et $300 \mathrm{~g}$ ( 9 points). En revanche, à partir de cette 
dernière dose, la digestibilité de la matière organique ( $\mathrm{dMO}$ ) de la ration totale ne progresse plus (fig. 1).

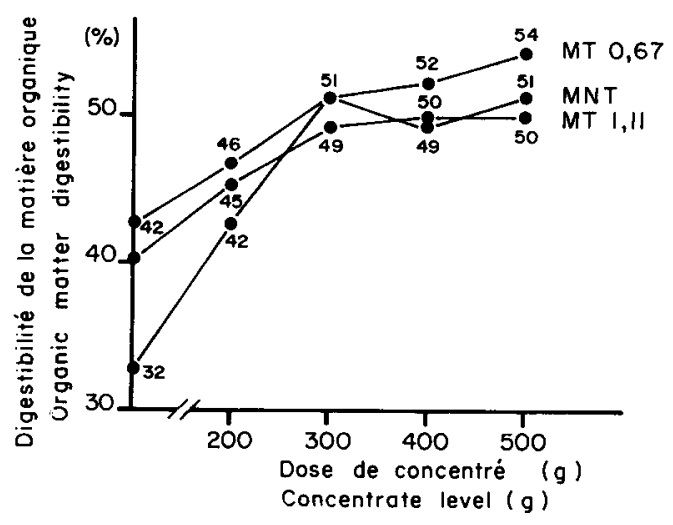

FIG. 1

Effet de la dose de concentré sur la digestibilité de la matière organique de la ration totale de marc.

Effect of the concentrate feed level

on the organic matter digestibility of grape marc diets.

2. Effet sur la digestibilité de la ration totale à base de marcs traités à la soude

Le traitement à la soude améliore la digestibilité de tous les constituants des marcs (tabl 2). En moyenne, celle des matières azotées passe de 10 à 25 p. 100 et celle de la matière organique de 32 à 42 p. 100. Cependant, la dose de 1,11 p. 100 de $\mathrm{NaOH}$ comparativement à la dose de 0,67 p. 100 , fait diminuer la digestibilité de la matière organique (dMO) et celle de l'azote, respectivement de 5 et de 18 p. 100 .

La complémentation des marcs traités entraine une plus faible augmentation de la dMO, comparativement aux résultats enregistrés pour les MNT. En effet, entre 0 et $500 \mathrm{~g}$ de concentré, l'accroissement de la dMO représente en moyenne 16 , 9 et 9 points respectivement pour MNT, MT 0,67 et MT 1,11.

\section{Effet du niveau de concentré sur la digestibilité propre des marcs}

La dMO du concentré calculée selon les données des tables I.N.R.A. (1978) s'établit à 82,1 p. 100 . En considérant ce niveau de digestibilité invariable quelle que soit la dose de concentré, nous avons estimé la digestibilité propre de la matière organique des marcs composant les différentes rations. Les résultats sont illustrés par la figure 2. Il apparaît que, la dMO propre des mares non traités progresse respectivement de $1,6,8,6,3,5$ et 2,4 points en présence de $200,300,400$ et $500 \mathrm{~g}$ de concentré. Cette augmentation s'explique par la fourniture d'azote aux microbes du rumen, elle passe par un maximum à $300 \mathrm{~g}$ de concentré (fig. 2). En revanche, lorsque les marcs sont traités, leur digestibilité en présence des mêmes doses de concentré est peu modifiée (fig. 2) car le traitement à la soude avait déjà fourni l'azote qui manquait aux microbes du rumen. 


\section{TABLEAU 2}

Effet de différents niveaux de concentré

sur la digestibilité de rations à base de marcs de raisin traités ou non à la soude.

Effect of the level of concentrate feeds

on the apparent digestibility of untreated and treated grape marc diets.

\begin{tabular}{|c|c|c|c|c|c|c|}
\hline & \multirow{2}{*}{$\begin{array}{c}\text { Digestibilité }(\%) \\
\text { Apparent } \\
\text { digestibility }(\%)\end{array}$} & \multicolumn{5}{|c|}{$\begin{array}{l}\text { Niveau de concentré }(\mathrm{g}) \\
\text { Concentrate level }(\mathrm{g})\end{array}$} \\
\hline & & $\begin{array}{c}0 \\
0 \text { p. } 100\end{array}$ & $\begin{array}{l}200 \\
15 \text { p. } 100\end{array}$ & $\begin{array}{l}300 \\
21 \text { p. } 100\end{array}$ & $\begin{array}{c}400 \\
26 \text { p. } 100\end{array}$ & $\begin{array}{l}500 \\
31 \text { p. } 100\end{array}$ \\
\hline \multirow{4}{*}{ MNT } & $\begin{array}{l}\text { Matière organique } \\
\text { Organic matter }\end{array}$ & $31,8 \pm 3,3$ & $42,2 \pm 1,9$ & $50,6 \pm 4,5$ & $49,2 \pm 1,7$ & $50,8 \pm 1,4$ \\
\hline & $\begin{array}{l}\text { Matières azotées ... } \\
\text { Crude protein }\end{array}$ & $9,7 \pm 2,3$ & $40,8 \pm 1,8$ & $46,7 \pm 4,3$ & $48,1 \pm 1,4$ & $47,4 \pm 1,8$ \\
\hline & Cellulose Weende & $31,7 \pm 3,2$ & $42,0 \pm 2,7$ & $43,1 \pm 8,1$ & $31,1 \pm 2,5$ & $38,1 \pm 10,1$ \\
\hline & $\begin{array}{l}\text { Matière grasse } \cdots \\
\text { Ether extract }\end{array}$ & $46,0 \pm 2,0$ & $55,7 \pm 2,7$ & $56,3 \pm 6,6$ & $54,8 \pm 2,0$ & $58,1 \pm 4,9$ \\
\hline \multirow{4}{*}{$\begin{array}{l}\text { MT } \\
0,67\end{array}$} & $\begin{array}{l}\text { Matière organique } \\
\text { Organic matter }\end{array}$ & $41,9 \pm 3,1$ & $45,8 \pm 2,9$ & $50,9 \pm 2,4$ & $52,0 \pm 3,1$ & $53,6 \pm 4,0$ \\
\hline & $\begin{array}{l}\text { Matières azotées .. } \\
\text { Crude protein }\end{array}$ & $27,3 \pm 1,9$ & $36,4 \pm 3,7$ & $56,4 \pm 3,1$ & $54,4 \pm 3,6$ & $54,4 \pm 1,8$ \\
\hline & Cellulose Weende & $51,3 \pm 2,7$ & $53,1 \pm 2,4$ & $55,6 \pm 4,5$ & $57,7 \pm 2,3$ & $55,6 \pm 3,1$ \\
\hline & $\begin{array}{l}\text { Matière grasse } \\
\text { Ether extract }\end{array}$ & $63,1 \pm 2,1$ & $64,9 \pm 3,1$ & $73,5 \pm 5,6$ & $75,7 \pm 7,6$ & $68,8 \pm 2,5$ \\
\hline \multirow{4}{*}{$\begin{array}{l}\text { MT } \\
1,11\end{array}$} & $\begin{array}{l}\text { Matière organique } \\
\text { Organic matter }\end{array}$ & $39,8 \pm 2,1$ & $44,9 \pm 3,6$ & $48,7 \pm 3,0$ & $49,8 \pm 2,1$ & $50,4 \pm 2,0$ \\
\hline & $\begin{array}{l}\text { Matières azotées .. } \\
\text { Crude protein }\end{array}$ & $22,4 \pm 1,1$ & $23,1 \pm 2,9$ & $49,2 \pm 1,5$ & $48,2 \pm 3,1$ & $43,6 \pm 1,4$ \\
\hline & Cellulose Weende & $43,3 \pm 2,1$ & $52,3 \pm 2,7$ & $40,9 \pm 3,1$ & $42,2 \pm 2,1$ & $43,1 \pm 2,8$ \\
\hline & $\begin{array}{l}\text { Matière grasse } . . . \\
\text { Ether extract }\end{array}$ & $65,2 \pm 2,3$ & $65,7 \pm 3,8$ & $61,0 \pm 2,1$ & $74,1 \pm 5,1$ & $62,8 \pm 1,6$ \\
\hline
\end{tabular}




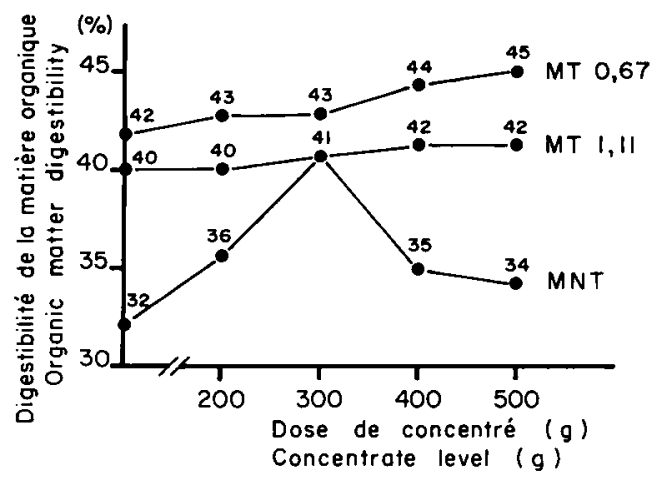

FIG. 2

Effet de la dose de concentré sur la digestibilité de la matière organique propre des marcs traités ou non à la soude.

Effect of the concentrate feed level on organic matter digestibility of untreated and treated grape marc.

\section{Discussion}

A. Faciès fermentaire des marcs de raisin traités ou non à la soude

Le dosage des produits volatils des ensilages des mares de raisin ( $\mathrm{AGV}, \mathrm{NH}_{3}$, alcools) révèle une modification du faciès fermentaire (traduisant une dégradation de la qualité) sous l'action de la soude.

La production de $\mathrm{C} 4$ est multipliée par 10 lorsque la dose de soude passe de 0 à 1,11 p. 100 de la MS ; par ailleurs, pour cette dernière dose il y a apparition d'acide propionique qui indique une fermentation des protéines (Sauvant \& Gouet, 1979).

Ces résultats sont en accord avec ceux de Shultz, Ralston \& Shultz (1974) et ceux de Larwence, Hammouda \& Gaouas (1983) qui ont travaillé respectivement sur ensilage de paille de céréale et de marcs de raisin préalablement traités à la soude.

Les fortes productions d'AGV enregistrées occasionnent des pertes sensibles de matière sèche à l'étuve. Nous notons en effet, une différence de 2,5 points de matière sèche entre MNT et MT 1,11. L'équation de Sсносн (1949) modifiée par l'Institut Technique des céréales et des fourrages (MosNier \& PAPIN, 1978) pour la prise en compte à 100 p. 100 des alcools, permet de corriger la MS de façon satisfaisante (tabl. 1).

De cette forte production d'AGV (notamment de $\mathrm{C} 4$ ), nous pouvions nous attendre à une teneur plus élevée des ensilages en ammoniac. Mais, bien qu'un accroissement soit constaté parallèlement au niveau de traitement à la soude, la quantité d'ammoniac ne dépasse pas 4 p. 100 de l'azote total (tabl. 1); la fermentation 
butyrique affecterait donc très peu les matières azotées des marcs. Il en résulte que la teneur en ammoniac d'un ensilage de marc de raisin n'est pas un bon critère d'appréciation de sa qualité comme elle l'est pour les ensilages d'herbe.

\section{B. Influence du traitement à la soude sur la digestibilité des marcs de raisin}

Après traitement des marcs de raisin à la soude, la dMO s'est accrue de 10 points et de 8 points respectivement pour MT 0,67 et MT $1,11(\mathrm{P}<0,01)$. Cette augmentation est comparable à celle enregistrée pour les pailles de céréale (IsTASSE et al., 1981 ; Dulphy, Kouassi \& Bien-Aimé, 1982).

Pour la paille de céréale, le traitement à la soude désorganise très légèrement la lignine, affecte les hémicelluloses et à moindre degré la cellulose (RIQUET, 1979). La cristallinité de la cellulose diminue et les espaces interfibrillaires qui mesurent selon FAN, Lee \& BeARdMore (1980) environ 40 \& sont agrandis ce qui favorise la pénćtration des bactéries cellulolytiques; il en résulte donc une augmentation de la digestibilité du produit. Toutes ces modifications sont observées pour des doses de soude supérieures à $4 \mathrm{~g} / 100 \mathrm{~g}$ de MS (JACKSON, 1977).

Pour les marcs de raisin, la digestibilité maximum est obtenue avec des doses de soude très faibles $(0,67 \mathrm{~g}$, par $100 \mathrm{~g}$ de MS de marc) ; à ce niveau de traitement, l'action de la soude sur les composés pariétaux (75 p. 100 de la MS) est probablement nulle. La soude doit agir d'une part sur la disponibilité de l'azote puisque la digestibilité de celui-ci passe de 10 à 27 p. 100 respectivement pour MNT et pour MT 0,67 (cette plus grande disponibilité de l'azote aurait favorisé la digestibilité des autres constituants du produit) et d'autre part sur les tanins condensés ( 5 p. 100 de la MS) qui sont probablement oxydés et particulièrement désactivés. La désactivation des tanins par traitement à la soude suivie d'une amélioration de la digestibilité a été observée pour d'autres aliments ; c'est le cas notamment du sorgho grain (RE1Chert, Flemming \& Schwab, 1980) et des graines de Shotea Robusta (Panda, JAYARAM \& RAMAMURTHY, 1969).

La diminution de la dMO enregistrée pour MT 1,11 confirme nos précédents résultats (LARWence, Hammouda \& Gaouas, 1983); elle ne s'explique pas par la quantité de soude ingérée car elle s'élève seulement à $9 \mathrm{~g}$ par jour et par animal alors que des moutons consommant de la paille traitée à 5 p. 100 de soude peuvent en ingérer jusqu'à $50 \mathrm{~g}$ sans que la digestibilité de la ration soit significativement affectée (REXEN \& ThOMSEN, 1976). Il faut donc admettre que sous l'action de fortes doses de soude (supérieures à 1 p. 100 environ par rapport à la MS), il y a formation de substances inhibitrices de l'activité microbienne; les composés phénoliques, plus particulièrement les tanins peuvent être en effet oxydés en milieu alcalin en des formes quinoniques (THOMSON, 1964) très toxiques qui inhibent les enzymes bactériennes par suite de réactions d'addition avec les groupements thiols ou aminés de ces enzymes (Paris \& Moyse, 1976). A ce sujet, les résultats obtenus par LaRwence (1983) sont assez révélateurs. Ainsi, la synthèse de protéines microbiennes en rumen artificiel et en présence de $20 \mathrm{ml}$ d'extrait de MNT, MT 0,67 et MT 1,11 s'établitelle respectivement à 42,49 et $43 \mathrm{mg}$ par $100 \mathrm{ml}$ de milieu d'incubation (la valeur 49 étant significativement différente de 42 et 43 ).

Ces résultats expliquent probablement la diminution de 8 points de la dMO des marcs observés par Messaoudi (1982) lorsqu'ils sont traités à raison de $3 \mathrm{~g}$ de scude par $100 \mathrm{~g}$ de MS. 


\section{Effet du niveau de concentré sur la digestibilité totale des rations et sur la digestibilité propre des marcs}

Pour calculer la dMO des marcs, la connaissance de celle du concentré est nécessaire. Plusieurs méthodes peuvent être adoptées :

1) utiliser pour chacun des composants du concentré, la valeur de la dMO donnée par les tables de valeur nutritive des aliments (par exemple, les tables I.N.R.A. 1978) :

2) appliquer la méthode dite de différence ou celle des systèmes de 2 équations préconisée par GaSNIER \& VACHEL (1952);

3) utiliser la méthode par régression très récemment actualisée par GigER \& SaUVant (1983) ; elle suppose cependant pour être appliquée de façon simple et dans de bonnes conditions, la linéarité de la dMO de la ration totale en fonction de la dose de concentré ce qui, ici, n'est pas le cas (fig. 2).

Nous avons préféré les valeurs données par les tables I.N.R.A. (1978). Ceci revient à faire l'hypothèse que la dMO du concentré est invariable quel que soit son niveau dans la ration (ce qui doit être globalement le cas puisque, DulPHY et al. (1983) n’ont pas observé de différences de digestibilité de l'amidon du concentré lorsque le taux de ce dernier dans la ration varie entre 15 et 45 p. 100). Dans ces conditions, les calculs montrent que la dMO propre des marcs (lorsque la dose de concentré passe de 200 à $500 \mathrm{~g}$ ) ne varie pas de la même façon selon que le marc est traité ou non.

\section{Pour les marcs non traités}

Lorsque les marcs ne sont pas traités, leur digestibilité décrit une courbe curvilinéaire dont le maximum (41 p. 100 de dMO) est atteint pour la dose de $300 \mathrm{~g}$ de concentré. Puis, la dMO propre des marcs diminue pour s'établir à 34 p. 100 pour la dose de $500 \mathrm{~g}$ (fig. 2). La première et la deuxième partie de la courbe indiquent respectivement la manifestation d'interactions digestives positives et négatives.

Ces dernières sont gênantes dans la mesure où elles entraînent une diminution de la dMO de la ration totale : selon de nombreux auteurs (HenNing et al., 1980 ; Dulphy, Kounssi \& Bien-Aimé, 1982), elles apparaissent lorsque la dose de concentré augmente dans la ration (en général supérieure à $30 \mathrm{p} .100$ ); elles résultent de variations de l'équilibre amidon-paroi de la ration qui occasionnent une diminution du $\mathrm{pH}$ du contenu ruminal. Ainsi, le $\mathrm{pH}$ de l'inoculum de rumen passe-t-il de 6,63 (MNT) à $6,03,5,97$ et 5,88 respectivement pour la dose de concentré de 300,400 et $500 \mathrm{~g}$. Or, comme le rapportent Kincaid, Hoover \& Junkins, 1981 ; Hoover, Crawford \& Stern, 1982, la digestibilité des parois (composants essentiels des marcs) diminue lorsque le pH du contenu du rumen est inférieur à 6,0-6,5.

\section{Pour les marcs traités}

Lorsque les marcs sont traités à la soude, la dMO de la ration totale augmente dans le même sens que la dose de concentré. Elle passe de 46 à 54 p. 100 et de 45 à 50 p. 100 respectivement pour MT 0,67 et MT 1,11 lorsque le niveau de concentré varie entre 200 et $500 \mathrm{~g}$. Ces valeurs de digestibilité correspondent globalement au maintien de la dMO du concentré $(82$ p. 100$)$ et des marcs (respectivement 42 et 40 p. 100 pour MT 0,67 et MT 1,11). Il en résulte donc, qu'il n'y aurait pas d'interactions digestives importantes entre marcs traités et niveau de concentré, contrairement à ce que nous avons observé pour MNT (fig. 2). 
La mesure du $\mathrm{pH}$ du contenu du rumen pour MT 0,67 montre que le $\mathrm{pH}$ est respectivement de $6,34,6,44$ et 6,22 pour la dose de 300,400 et $500 \mathrm{~g}$ de concentré. Ces valeurs de $\mathrm{pH}$ sont probablement propices à la dégradation maximale à la fois des marcs et du concentré. Il est alors fort probable que potentiellement et, dans les conditions classiques d'alimentation des animaux, la dMO propre des marcs ne puisse dépasser environ 42 p. 100.

Ce niveau de digestibilité peut être obtenu soit par une complémentation azotée et énergétique, soit par une complémentation avec de l'urée $(12 \mathrm{~g} / \mathrm{kg}$ de MS de marc) soit par un traitement à la soude à la dose de 0,67 p. 100 par rapport à la MS.

Dans ces conditions, il ne semble pas très avantageux du point de vue économique de complémenter les marcs de raisin préalablement traités à la soude à la dose de 0,67 p. 100 lorsqu'ils sont offerts à des animaux à l'entretien ou à faible production.

En effet, la quantité de matière organique digestible ingérée pour des moutons pesant entre 40 et $45 \mathrm{~kg}$, s'établit à 393,513 et $483 \mathrm{~g}$ (respectivement pour MNT, MT 0,67 et MT 1,11) pour une distribution de $1300 \mathrm{~g}$ de MS de marc en l'absence de complémentation contre 540,538 et $509 \mathrm{~g}$ pour une distribution de $860 \mathrm{~g}$ de MS de marc plus $300 \mathrm{~g}$ de concentré.

\section{Conclusion}

Le traitement des marcs de raisin avec une faible dose de soude $(0,67$ p. 100/MS) améliore de 10 points la dMO (elle passe de 32 à 42 p. 100).

La complémentation azotée et énergétique de ces marcs, n'exerce pas la même action sur la dMO du produit selon qu'il est traité ou non.

Lorsque les marcs ne sont pas traités, la dMO de la ration totale augmente jusqu'à la dose de $300 \mathrm{~g}$ de concentré, puis reste invariable pour les doses de $400 \mathrm{et}$ $500 \mathrm{~g}$.

Lorsque les marcs sont traités, la dMO de la ration totale augmente parallèlement à la dose de concentré. Elle passe de 42 à 54 p. 100 pour MT 0,67 ce qui correspond globalement au maintien de la dMO propre des marcs. Il n'y aurait donc pas après traitement, d'interactions digestives significatives, contrairement à ce qui est observé pour les mares non traités.

Il semble par ailleurs que la digestibilité potentielle des marcs dans les conditions classiques d'alimentation ne dépasse pas $40-42$ p. 100 . Ce niveau de digestibilité peut être obtenu soit par une complémentation azotée et énergétique $(300 \mathrm{~g}$ de concentré pour $1000 \mathrm{~g}$ de MS de marc) soit par une complémentation en azote non protéique $(12 \mathrm{~g}$ d'urée $/ 1000 \mathrm{~g}$ de $\mathrm{MS}$ de marc) soit par un traitement à la soude $(0,67 \mathrm{p} .100 /$ MS).

Reçu en avril 1985.

Accepté en juillet 1985. 


\section{Summary \\ Nutritive value of grape marc}

IV. Effect of different levels of concentrate feeds on the digestibility of sodium hydroxide treated or untreated grape marc diets in sheep

With the aim of determining the optimum level of concentrate feeds based on rapeseed oil meal leading to a maximum digestibility of sodium hydroxide treated or untreated grape marc silage $(0.67$ and $1.11 \%$ relative to dry matter -DM), we used groups of 4 adult sheep to measure the digestibility of diets containing $200,300,400$ and $500 \mathrm{~g}$ concentrate, i.e. 15 , 21, 26 and 31 p. 100, respectively of the total diet relative to DM.

Grape marc may be satisfactorily stored in the form of silage. When treated with sodium hydroxide, the production of butyric acid increased by 12 points for the $1.11 \mathrm{p} .100$ treatment without changing the ammonia production (table 1).

Organic matter digestibility (OMd) increased as affected by the treatment; it was 32,42 and 40 p. 100, respectively for $0,0.67$ and 1.11 p. 100 sodium hydroxide (table 2).

Protein and energy supplementation of these three categories of marc did not have the same effect on OMd of the total diet and specifically on grape marc, according as the product was treated or not (fig. 1 and 2).

When untreated marc was used, OMd of the total diet reached a maximum when adding $300 \mathrm{~g}$ concentrate feeds whereafter it remained unchanged due to the specific marc OMd decrease from 41 to 35 and 34 p. 100, respectively for concentrate levels of 300,400 and $500 \mathrm{~g}$.

With treated marc, OMd of the total diet increased parallel to the amount of concentrate feed used. For instance, when using 0.67 p. 100 sodium hydroxide, OMd of the total diet increased from 42 to 54 p. 100 , corresponding to the maintenance of the specific oMd of the treated grape marcs. Accordingly, opposite to untreated marcs there did not seem to be any significant digestive interactions in the case of treated marcs.

Key words : Grape marc silage, concentrate feed level, sodium hydroxide treatment, sheep digestibility.

\section{Références bibliographiques}

Conway E.J., 1965. Micro diffusion analysis and volumetric error. $5^{\circ}$ ed. Crosby Lockwood, London, $468 \mathrm{pp}$.

Dulphy J.P., Kouassi A., Bien-Aimé A., 1982, Etude de la valeur alimentaire des pailles de céréales traitées ou non à la soude. II. Influence de la nature du complément énergétique. Ann. Zootech., 31, 215-232.

Dulphy J.P., Breton J., Louyot J.M., Bien-Aimé A., 1983. Etude de la valeur alimentaire des pailles traitées ou non à la soude. III. Influence du niveau d'apport de l'aliment concentré. Ann. Zootech., 32, 53-80.

FABre J., 1909. Sur la valeur alimentaire du marc de raisin. Ann. de L'E.N.A. de Montpellier. Fasc. III, 219-228.

Fan L.T., Lee Y.H., Beardmore H., 1980. Major chemical and physical features of cellulosic materials as substrates for enzymatic hydrolysis Adv. in Biochem. Eng., 14, 101-116.

Gasnier A., Vachel J.P., 1952. Recherche sur la méthode de mesure de la digestibilité d'un aliment : condition d'emploi de cette méthode. Ann. Zootech., 1, 157-174.

Giger S., Sauvant D., 1983. Comparaison de différentes méthodes d'évaluation du coefficient d'utilisation digestive des aliments concentrés par le ruminant. Ann. Zootech., 32, 215-246. 
Hammouda F., 1984. Effet de différents traitements chimiques sur les composés pariétaux des marcs de raisin et sur leur digestibilité. Thèse de Magister, 91 p. I.N.A. El-Harrach, Algérie.

Henning P.A., Van Derlinden Y., Mattheyse M.E., Nauhaus W.K., Schwartz H.M., 1980. Factors affecting the intake and digestion of roughage by sheep fed maize straw supplemented with maize grain. J. Agric. Sci. Camb., 94, 565-573.

Hoover W.H., Crawford R.J., Stern M.D., 1982. Effect of solids and liquids flows on fermentation in continuous cultures. III. Solids retention time. J. Anim. Sci., 54, 849-854.

I.N.R.A., 1978. Alimentation des ruminants. Ed. 1.N.R.A. Route de Saint-Cyr, 78000 Versailles, $597 \mathrm{p}$.

Istasse L., Van Eenaeme C., Lambot O., Gielen M., Bienfait J.M., 1981. Etude de quelques facteurs de variations de la digestibilité in vitro. Application à un foin traité ou non à la soude. Ann. Zootech., 30, 183-195.

JACKSON M.G., 1977. The alkali treatment of straw. Anim. Feed Sci. Technol., 2, 105-130.

Kincaid C.R., Hoover W.H., Junkins L.L., 1981. The effect of pH and dilution rate on rumen fermentation in continuous culture. J. Anim. Sci., 53 (Abstr.) p. 411.

LARWENCE A., 1983. Recherches sur les facteurs limitants de la faible valeur nutritive des marcs de raisin pour le ruminant : procédés d'amélioration. Thèse de Doctorat ès sciences naturelles, 248 p. Université de Dijon.

Larwence A., Yahiaour A., 1983. Valeur alimentaire des marcs de raisin. I. Influence de 8 sources azotées de complémentation sur l'utilisation digestive, par le mouton, de marc de raisin épuisé et ensilé. Ann. Zootech., 32, 357-370.

Larwence A., Hammouda F., GaOuas Y., 1983. Valeur alimentaire des marcs de raisin. II. Effet d'un traitement à la soude sur la valeur alimentaire, chez le mouton, de marc de raisin épuisé à la vapeur et ensilé. Ann. Zootech., 32, 371-382.

MESSAOUdi L., 1982. Essai d'amélioration de la valeur alimentaire du marc de raisin ensilé. Mémoire de fin d'études, 82 p. E.N.S.S.A.A., Dijon.

Mosnier M., Papin C., 1978. Qualité de conservation des ensilages et bilan en silo, Perspectives agricoles, 15, 80-89.

Panda B., Jayaram M., Ramamurthy N.S., 1969. Processing and utilization of salseed (Shorea robusta) as a source of energy in poultry feed. Indian Vet. J., 46, 1673-1682.

Paris R.R., Moyse H., 1976. Précis de matière médicale, 309 p. Masson ed., Paris.

Reichert R.D., Fleming S.E., Schwab D.J., 1980. Deactivation and Nutritional improvement of sorghum by anaerobic storage of $\mathrm{H}_{2} \mathrm{O}, \mathrm{Hcl}$ or $\mathrm{NaOH}$ treated grain. J. Agric. Fd. Chem., 28, 824-831.

ReXen F., Thomsen K.V., 1976. The effect on digestibility of a new technique for alkali treatment of straw. Anim. feed Sci. Technol., 1, 73-86.

Rigaud J., Journet M., 1970. Méthode de dosage des acides gras volatils dans le liquide du rumen. Ann. Biol. Anim. Bioch. Biophy., 10, 151-157.

RiqueT A.M., 1979. Etude des constituants membranaires de divers produits végétaux destinés à l'alimentation animale : signification et limites des principales méthodes de dosage. Thèse de Doctorat de $3^{\circ}$ cycle. Université de Paris.

Sauvant D., Gouet P., 1979. Les relations entre les processus fermentaires : Conséquences pour l'appréciation quantitative de la qualité de la conservation. In : La conservation des ensilages. C.A.A.A., INA Paris-Grignon, pp. 41-77.

SсHосн W., 1949. Die bei der trocknung von selageprobein im trockenschrank auftretender verluste von flüchtingen saüren und basen. Mitteilungen aus dem Gebiete der Lebensmitteluntersuchung und hygiene, 40, 179-189.

Shultz T.A., Ralston A.T., Shultz E., 1974. Effect of various additives on nutritive value of ryegrass straw silage. I. Laboratory silo and in vitro dry matter digestion observations. J. Anim. Sci., 39, 920-925.

Tномson R.S., 1964. Structure and reactivity of phenolic compounds. In : Biochemistry of phenolic compounds, pp. 1-32 H.B. HARBon ed. Acad. Press. London. 